\title{
Experimental Analysis of Open-Circuit Voltage Hysteresis in Lithium-Iron-Phosphate Batteries
}

\author{
F. Baronti*, W. Zamboni ${ }^{\S}$, N. Femia ${ }^{\S}$, R. Roncella*, and R. Saletti* \\ *Dipartimento di Ingegneria dell'Informazione, Università di Pisa, I-56122 Pisa, Italy \\ e-mail: f.baronti@iet.unipi.it \\ $\S$ Dipartimento di Ingegneria dell'Informazione, Ingegneria Elettrica e Matematica Applicata (DIEM) \\ Università degli Studi di Salerno, I-84084 Fisciano (SA), Italy \\ e-mailwzamboni@unisa.it
}

\begin{abstract}
This paper aims at investigating and modelling the hysteresis in the relationship between state-of-charge and opencircuit voltage of lithium-iron-phosphate batteries. A first-order charge relaxation equation was used to describe the hysteresis dynamics. This equation was translated into a voltage-controlled voltage source and included within an equivalent electric circuit of the battery used in online state-of-charge estimators. The effectiveness of the obtained battery model was verified comparing simulated and experimental data.
\end{abstract}

\section{INTRODUCTION}

Lithium-ion (Li-ion) batteries offer the best trade-off between power/energy density and costs for energy storage in Electric Vehicles (EVs) and Hybrid Electric Vehicles (HEVs). A great deal of research on this technology has led to the introduction of different chemical compositions of the battery, which differ in the materials of the electrodes and the electrolyte and thus in the achieved performances [1]-[3].

The variant using an iron-based cathode (e.g., lithiumiron-phosphate, $\mathrm{LiFePO}_{4}$ ) is one of the most promising for EV/HEV applications. $\mathrm{LiFePO}_{4}$ batteries are safer and cheaper than those based on lithium cobalt oxide cathode and its evolutions, which partly replace the Cobalt with Nickel-ManganeseCobalt (NMC) or Nickel-Cobalt-Aluminum (NCA) [3]. They are characterised by a lower operating voltage $(2.5-3.6 \mathrm{~V})$ and a flat Open-Circuit Voltage (OCV) with State-of-Charge (SoC) ranging from $20 \%$ to $80 \%$. Moreover, they exhibit a pronounced hysteresis [4]-[6].

For a reliable operation of EVs/HEVs, a Battery Management System (BMS) must evaluate the current amount of energy stored in the battery, its power capability and health [7]-[10]. This task, in turn, demands the online estimation of the SoC and the electric parameters of the battery [11]-[14]. This can only be achieved by means of an accurate measure of the hysteresis characteristics and an affordable model of the hysteresis phenomenon.

First, this paper presents the Pulsed Current (PC) testing of a $20 \mathrm{~A} \mathrm{~h} \mathrm{LiFePO}_{4}$ cell at room temperature. Then, the hysteresis in the SoC-OCV plane is investigated in the stream of [4], [15]. The developed hysteresis model is then added as a voltage contribution to the output equation of the battery electrical model already used in [13], [16] for NMC Li-ion cells, obtaining a model suitable for online estimators. The effectiveness of the electrical model including hysteresis is finally verified with experimental results.

\section{Experimental Tests on a LiFePO ${ }_{4}$ Cell}

The experimental characterisation of a $\mathrm{LiFePO}_{4}$ cell to obtain the relationship between $\mathrm{OCV}$ and $\mathrm{SoC}$ requires a welldefined and reliable test procedure, as OCV is very sensitive to the way in which it is measured. First of all, we ruled out the possible dependence on room temperature variations by performing the experiments inside the thermal chamber Binder MK53, by which the cell's ambient temperature $T$ is kept constant at $T=25^{\circ} \mathrm{C}$. A Keithley 2420 SourceMeter provides the cell current (either as current source or current sink) and measures the cell current and voltage with a 4-wire connection to the cell. The SourceMeter is remotely controlled by a LabVIEW application developed ad hoc. The application controls the execution of the experiments, by configuring the instrument, acquiring the measurement values and saving them in a $\log$ file.

The experiment can freely be configured as a sequence of test steps described in a test description file. The test steps consist of charge, discharge and rest operations with configurable parameters. The charge and discharge steps are used to transfer a specified amount of charge to/from the cell at a constant current. The configuration parameters are the charging/discharging current values and the step duration (which determine the amount of charge transferred), as well as the target charging/discharging voltage. In this way, a step can end because the cell voltage reaches the target value or the current drops below a given percentage of the set value, thus implementing the constant current (CC) and the constant voltage (CV) phases for a full charge/discharge of the cell. The rest step introduces a pause between the steps in which the battery current is zero. The sampling rate of the cell current and voltage is $1 \mathrm{~Hz}$, for every experiment.

\section{A. Pulsed Current Testing}

The above described experimental test-bed provides an automatic and reliable framework for the characterisation of a cell, which usually is a rather long and time consuming task. In order to investigate the relationship between OCV and $\mathrm{SoC}, \mathrm{SoC}$ is changed with charge/discharge steps. The 


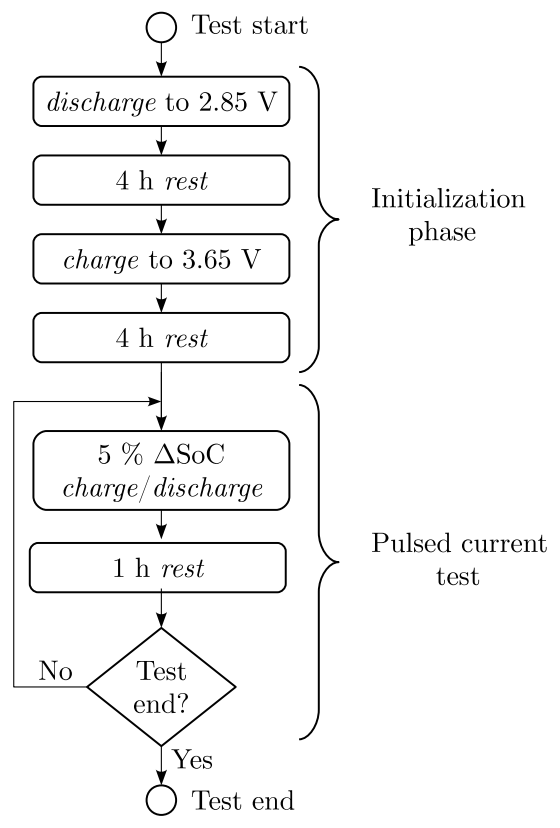

Fig. 1. Flow chart of a test execution.

variation $(\Delta \mathrm{SoC})$ of the charge stored in the cell is $5 \%$ of the nominal capacity for each step. This is obtained with a current pulse of $0.5 \mathrm{~h}$ duration and amplitude equal to $C / 10$ (where $C$ is the numerical value of the cell nominal capacity expressed in $\mathrm{Ah}$ ). Each charge/discharge is followed by a $1 \mathrm{~h}$ rest of the cell (rest step), as it is shown by the $1 \mathrm{~h}$ rest block in the flow diagram of Fig. 1, in order to extract the proper OCV value at the given $\mathrm{SoC}$ in steady-state conditions, i.e., when relaxation phenomena are reasonably vanished. OCV is thus defined as the cell voltage at the end of the $1 \mathrm{~h}$ rest.

Some Pulsed Current (PC) tests have been designed and carried out to investigate the dependence of the OCV on the way a given $\mathrm{SoC}$ value is reached and thus the hysteresis phenomena that occur in the cell under test. In more detail, three different experiments were defined, in which the SoC is varied over the three loops described in the flow diagrams of Fig. 2. The basic idea is to explore the hysteresis in three different discharge/charge loops of different sizes, each one included in the other. The major loop of the relationship between $\mathrm{OCV}$ and $\mathrm{SoC}$ is the complete discharge/charge cycle of the cell. It is labeled PC test (a) in the left-hand side of Fig. 2. The two inner loops are labeled PC test (b) and (c) and respectively span $50 \%$ and $20 \%$ of the SoC range. It is worth noting that each PC test is started with the cell in the "same" condition, which is reached after the proper Initialisation phase shown in Fig. 1. This phase includes a full battery discharge, a $4 \mathrm{~h}$ rest and a full recharge followed by another $4 \mathrm{~h}$ rest.

The cell under test is a brand-new $20 \mathrm{Ah} \mathrm{LiFePO}_{4}$ cell. The charge/discharge cut-off voltages are $3.65 \mathrm{~V}$ and $2.85 \mathrm{~V}$, respectively, as specified by the cell manufacturer. The cell was first conditioned by ten full charge/discharge cycles, after the delivery from the manufacturer.

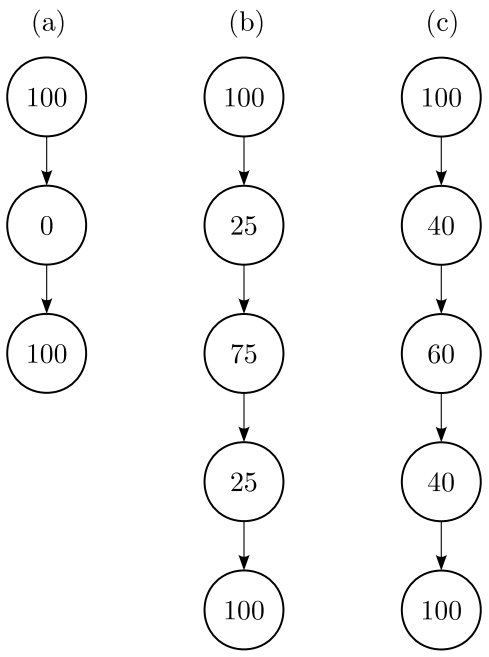

Fig. 2. SoC loops. Each SoC variation is obtained through the current pulses (with $C / 10$ amplitude and $1 \mathrm{~h}$ duration).

\section{B. Experimental Results}

The results of the experiments described by the flow diagrams in Fig. 2 are reported in Fig. 3 and Fig. 4. Let us define $Q(t)$ the charge stored in the battery cell at time $t$. If we start from a known value $Q_{0}$ of the charge at time $t_{0}$, $Q_{0}=Q\left(t_{0}\right)$, the charge $Q\left(t_{k}\right)$ at the generic time instant $t_{k}$ can be calculated by the mid-point integration of the sequence of the cell current samples $i\left(t_{k}\right)$ as:

$$
Q\left(t_{k}\right)=Q_{0}+\sum_{j=1}^{k} \frac{i\left(t_{j}\right)+i\left(t_{j-1}\right)}{2}\left(t_{j}-t_{j-1}\right)
$$

being the cell current $i$ positive during the charge process. We set the initial point of each experiment when the battery is fully charged, after the Initialisation phase $\left(Q_{0}=20 \mathrm{Ah}\right)$. This assumption is arbitrary and can lead to negative values of $Q(t)$, if the charge variation computed according to (1) during the $100 \%$ to $0 \%$ SoC discharge part of the PC test (a) is larger than the $20 \mathrm{Ah}$ nominal capacity for the given cell sample used in the experiments.

PC test (a) shows that the characteristic is rather flat as expected, with two particularly flat zones in the upper and in the lower parts of the curve. The first zone is around the mid charge value $(10 \mathrm{Ah})$, the second one is around $17.5 \mathrm{Ah}$. We also note the very pronounced hysteresis effect between the discharge and the charge curves. If we span the SoC from $2 \mathrm{Ah}$ to $18 \mathrm{Ah}$, i.e., we explore around $80 \%$ of the entire SoC range, the $\mathrm{OCV}$ variation is less than $0.2 \mathrm{~V}$, a value as small as $6 \%$ of the $3.3 \mathrm{~V}$ rated voltage. Such experimental evidence is in accordance with [4].

Fig. 4 shows the experimental results coming from PC test (b) and (c), i.e., the experiments in which the cell is stimulated with the two minor discharge/charge loops. The initial points of the three experiments, obtained after the cell Initialisation phase, are highlighted with an empty marker. It is important to remark that these points are almost identical and that the 


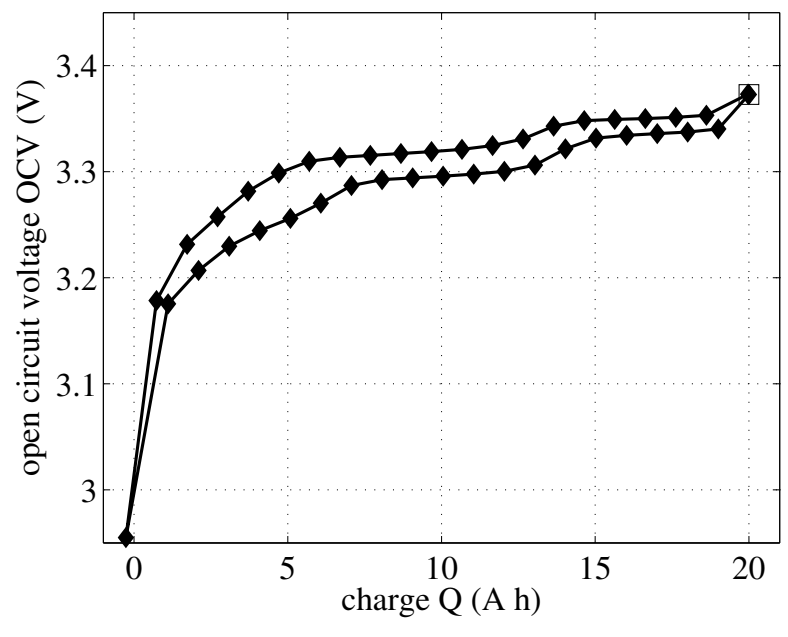

Fig. 3. Hysteresis major loop obtained by running the experiment (a) (see Fig. 2. Markers $(\diamond)$ indicate the experimental points obtained after $1 \mathrm{~h}$ relaxation time. The starting point of the experiment (after cell conditioning) is highlighted with an empty marker $(\square)$.

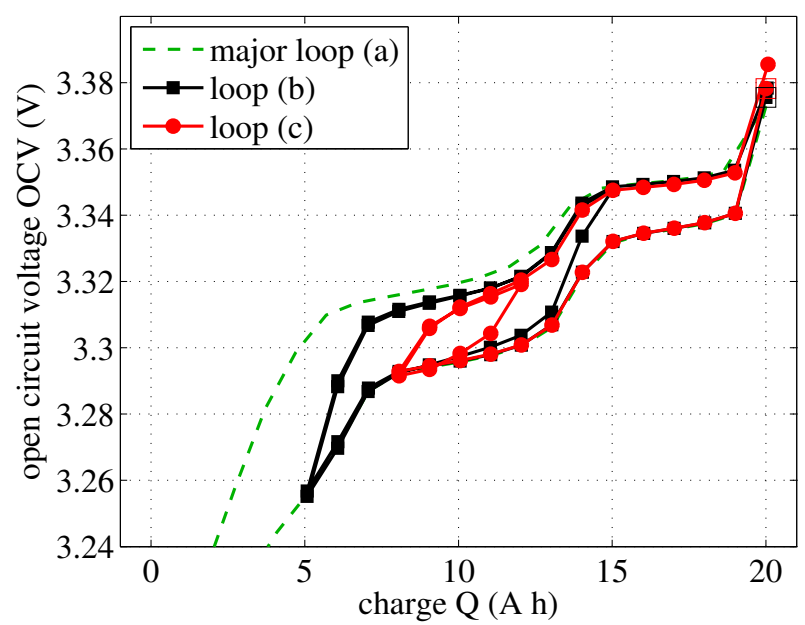

Fig. 4. Hysteresis minor loops. The starting point of the experiment (after cell conditioning) is highlighted with an empty marker $(\square)$ in each case.

three initial discharge curves almost overlap, showing the good repeatability of the experiment.

Another important result comes from the analysis of the hysteresis behaviour. The minor loops curves are "attracted" towards the bounds set by the major discharge/charge loop, but the attraction is completed only after a sort of "delay" in charge, after which the OCV values are almost indistinguishable from experiment to experiment. In other words, the upper and lower bounds are reached only with a "charge delay" after the point in which the charge or discharge process is inverted. A simple way to model this hysteresis phenomenon is to assume that the hysteresis is governed by a state equation that links the cell SoC to the OCV. The model we propose is described in the following section.

\section{Electrical Model of A LiFePO ${ }_{4}$ Cell including VOLTAGE HYSTERESIS}

Let us consider a cell having a nominal capacitance $Q_{\mathrm{n}}$ and let us call $v$ and $i$ the cell voltage and current, respectively, with the passive sign convention. We also assume the cell temperature constant and equal to the room temperature $T$. The aim of this section is to present a simple model of the hysteresis phenomena and to include it in an equivalent electrical model of a Li-ion cell (e.g. [13]) widely adopted in the literature.

\section{A. Charge Normalisation}

The cell SoC is defined by a linear transformation such that $\operatorname{SoC}\left(Q=Q_{n}\right)=1$ and $\operatorname{SoC}(Q=0)=0$.

\section{B. Hysteresis as a Function of State-of-Charge}

Before introducing the hysteresis model, let us make the following assumptions.

1) First, we assume that the hysteresis effects may be represented by an additive term $v_{\mathrm{H}}$ to the cell terminal voltage $v$.

2) The hysteresis term $v_{\mathrm{H}}$ is constant when the cell is at rest ( $i$ is zero). This means that every time-relaxation phenomenon is not included in the hysteresis model and must be modelled separately.

3) It is possible to define $v_{\mathrm{H}}$ as a function of the SoC history. Further, we introduce the maximum hysteresis loop which "contains" every evolution and thus trajectory in the plane $\mathrm{SoC}-v_{\mathrm{H}}$ (state-space for hysteresis). The maximum hysteresis loop is calculated from its upper bound $\mathrm{OCV}_{\mathrm{up}}(\mathrm{SoC})$ and its lower bound $\mathrm{OCV}_{\mathrm{lw}}(\mathrm{SoC})$. We also define the average open circuit voltage $\mathrm{OCV}_{\mathrm{av}}(\mathrm{SoC})$ as:

$$
\mathrm{OCV}_{\mathrm{av}}(\mathrm{SoC})=\frac{\mathrm{OCV}_{\mathrm{up}}(\mathrm{SoC})+\mathrm{OCV}_{\mathrm{lw}}(\mathrm{SoC})}{2} .
$$

The $\mathrm{OCV}_{\mathrm{av}}$ as defined in (2) is calculated from the experimental data and is plotted in Fig. 5. It is finally useful to define the non-negative function $E(\mathrm{SoC})$ representing the maximum deviation due to the hysteresis of the OCV value with respect to the average value $\mathrm{OCV}_{\text {av }}$

$$
E(\mathrm{SoC})=\frac{\mathrm{OCV}_{\mathrm{up}}(\mathrm{SoC})-\mathrm{OCV}_{\mathrm{lw}}(\mathrm{SoC})}{2},
$$

whose diagram is reported in Fig. 6.

As explained above, each SoC variation causes the hysteresis term $v_{\mathrm{H}}$ to vary with inertia. We use a first order relaxation equation to model the inertia, as it happens for the voltage relaxation in a $\mathrm{RC}$ circuit subject to a forcing voltage. We have to take into account in this case that SoC variations might be negative, whereas time variations are always positive in the time domain equations. With this idea in mind, we can write 


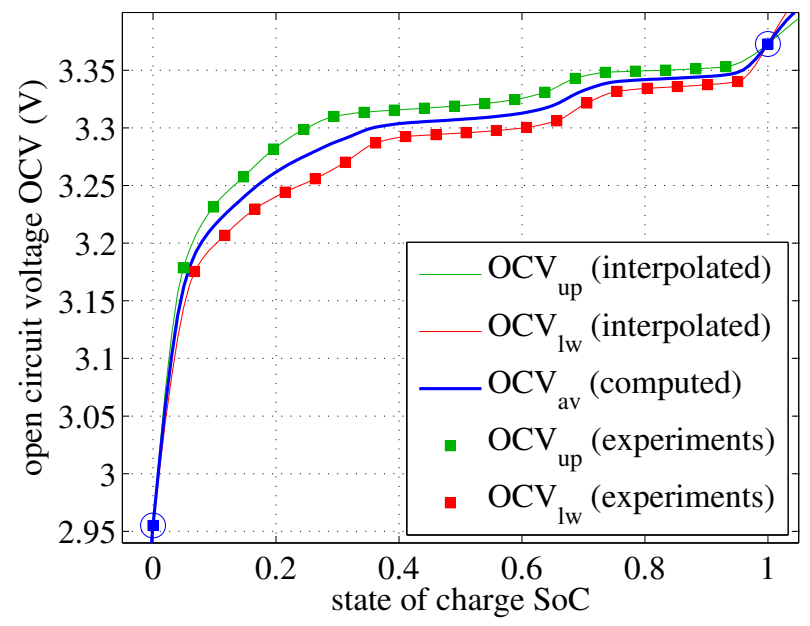

Fig. 5. Hysteresis major loop and average open circuit voltage defined by (2), as functions of SoC.

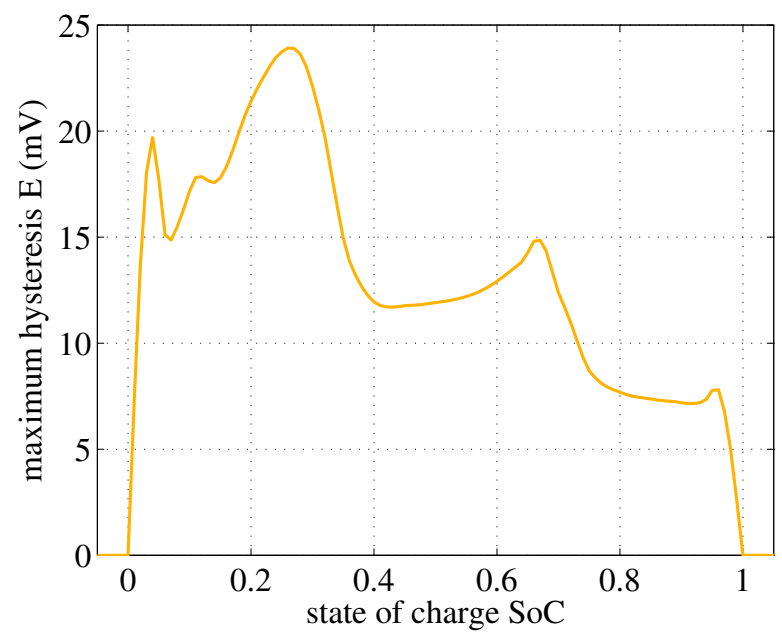

Fig. 6. Maximum positive hysteresis, defined according to (3) as a function of SoC (- solid orange line).

the differential equations describing the model in the $\mathrm{SoC}-v_{\mathrm{H}}$ plane as:

$$
\begin{array}{ll}
\frac{d v_{\mathrm{H}}}{d \mathrm{SoC}}=-\gamma v_{\mathrm{H}}+\gamma[+E(\mathrm{SoC})] & \text { for } d \mathrm{SoC} \geq 0 \\
\frac{d v_{\mathrm{H}}}{-d \mathrm{SoC}}=-\gamma v_{\mathrm{H}}+\gamma[-E(\mathrm{SoC})] & \text { for } d \mathrm{SoC}<0
\end{array}
$$

where $1 / \gamma$ is a sort of "charge constant" analogous to the time constant used in the time domain. Joining (4) and (5) we finally obtain:

$$
\frac{d v_{\mathrm{H}}}{d \mathrm{SoC}}=\operatorname{sgn}(d \mathrm{SoC}) \gamma v_{\mathrm{H}}+\gamma E(\mathrm{SoC})
$$

Equation (6) recasts in the time domain by multiplying both members by $\frac{d \mathrm{SoC}}{d t}$.

\section{Electrical Model}

The equivalent electrical model of a Li-ion cell that includes the hysteresis effect is shown in Fig. 7. It is based on a model
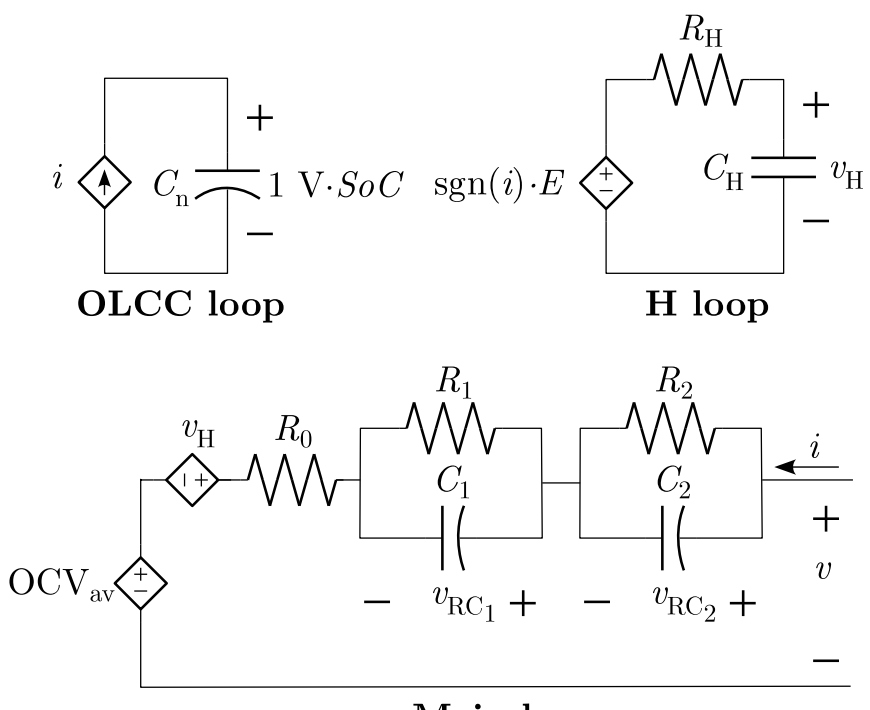

Main loop

Fig. 7. Cell circuit model

widely adopted in the literature [16] (the Main and the OLCC loops of Fig. 7) modified with an additional voltage source that accounts for hysteresis. The main loop models the output current-to-voltage characteristics:

$$
v=\mathrm{OCV}_{\mathrm{av}}+v_{\mathrm{H}}+R_{0} i+v_{\mathrm{RC} 1}+v_{\mathrm{RC} 2},
$$

in which $R_{0}$ is the series resistance of the cell, $v_{\mathrm{RC} k}$ are terms that account for the relaxation phenomena occuring also when $i=0$

$$
\frac{d v_{\mathrm{RC} k}}{d t}=-\frac{1}{R_{k} C_{k}} v_{\mathrm{RC} k}+\frac{i}{C_{k}},
$$

and $v_{\mathrm{H}}$ is the hysteresis term. The analysis described in this paper is carried out in consecutive steady-state points, so that we can safely assume $v_{\mathrm{RC} k}=0$.

The OLCC loop models the so-called open-loop Coulomb Counting (OLCC), governed by the equation

$$
i=Q_{\mathrm{n}} \frac{d \mathrm{SoC}}{d t}=\left(\frac{Q_{\mathrm{n}}}{1 \mathrm{~V}}\right) \frac{d}{d t}(\mathrm{SoC} \cdot 1 \mathrm{~V})=C_{n} \frac{d v_{\mathrm{SOC}}}{d t} .
$$

The state of charge (SoC) is represented by the "voltage" $v_{\mathrm{SOC}}$ on a capacitor $C_{n}=Q_{\mathrm{n}} / 1 \mathrm{~V}$ that ranges from 0 to $1 \mathrm{~V}$. Values below 0 and above 1 are acceptable (since these values are only reference points), but they show that the cell is going outside the desired operating range.

The hysteresis generator $v_{\mathrm{H}}$ contribution can be calculated by solving the time-domain couterpart of Eq.(6):

$$
\frac{d v_{\mathrm{H}}}{d t}=-\frac{v_{\mathrm{H}}}{\tau_{\mathrm{H}}}+\frac{E(\operatorname{SoC}(t))}{\tau_{\mathrm{H}}},
$$

where $\tau_{\mathrm{H}}=R_{\mathrm{H}} C_{\mathrm{H}}, C_{\mathrm{H}}=1 \mathrm{~F}$ and

$$
R_{\mathrm{H}}=\frac{Q_{\mathrm{n}}}{\gamma|i|}
$$

The electric circuit that implements (10) and calculates the $v_{\mathrm{H}}$ value is the $\mathrm{H}$ loop in Fig. 7. We note that $R_{\mathrm{H}}$ behaves as an 

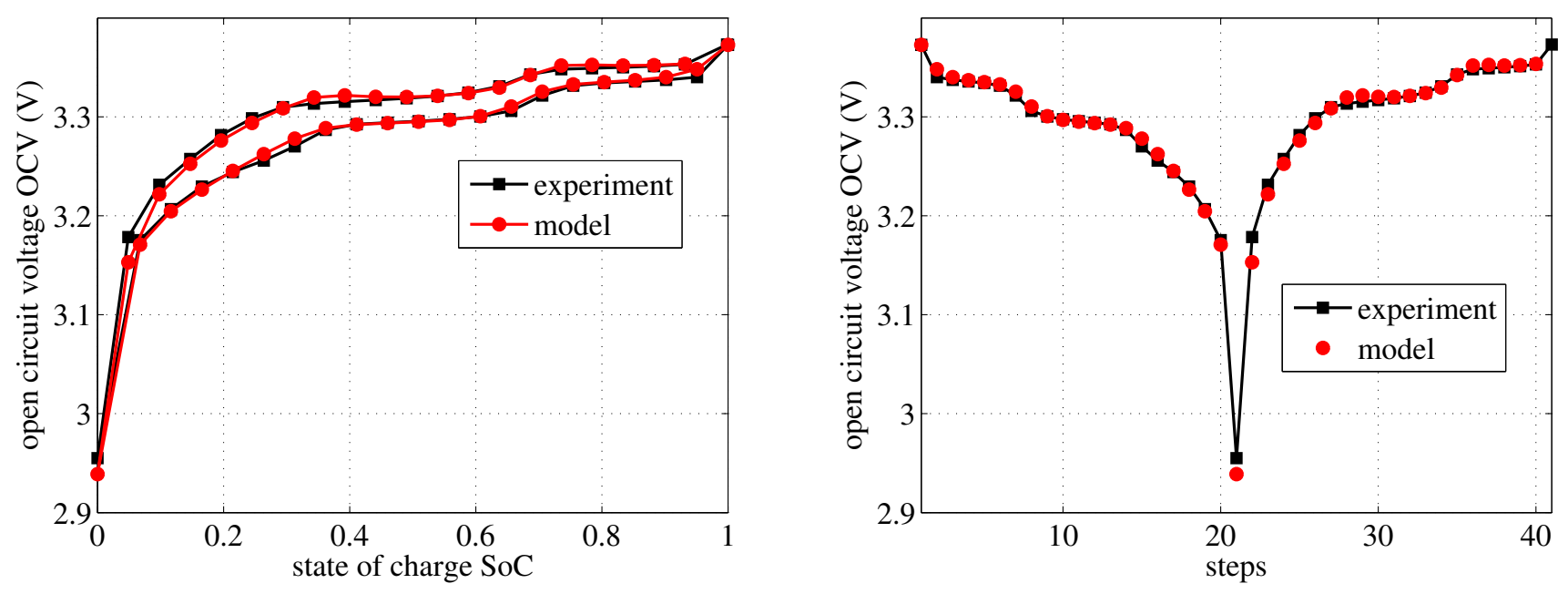

(a) Pulsed current test (for description see Fig. 2)
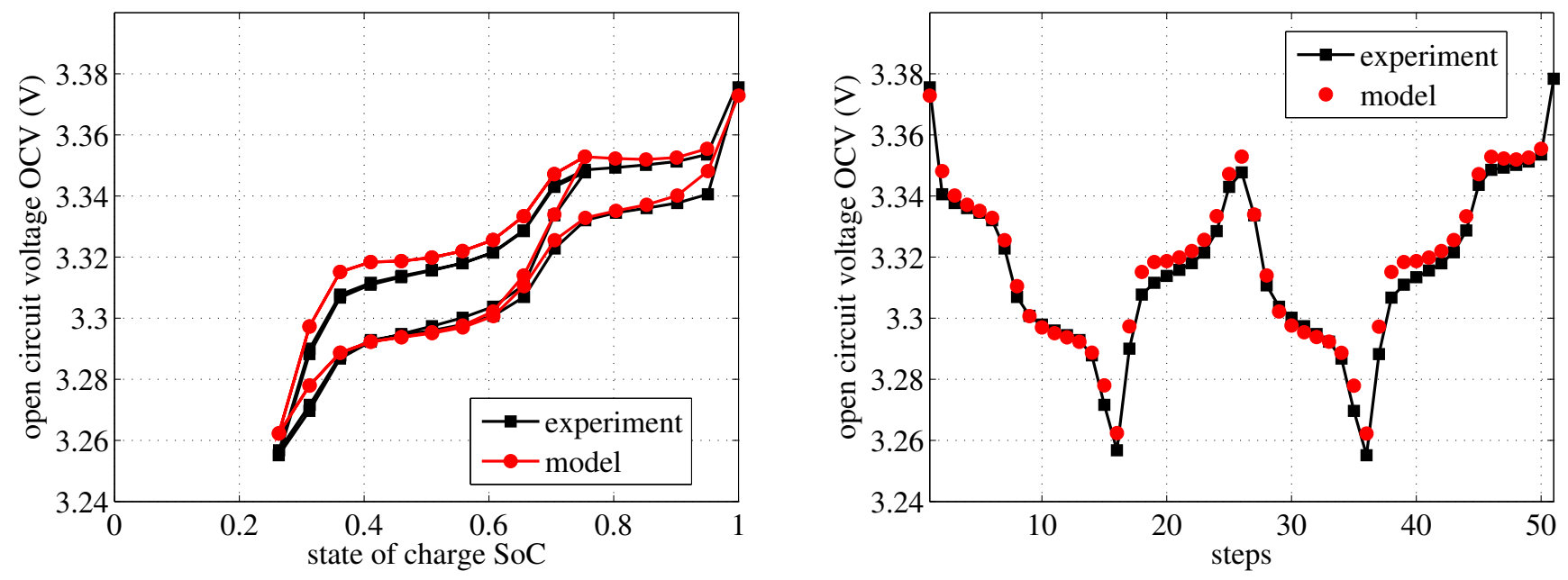

(b) Pulsed current test (for description see Fig. 2)
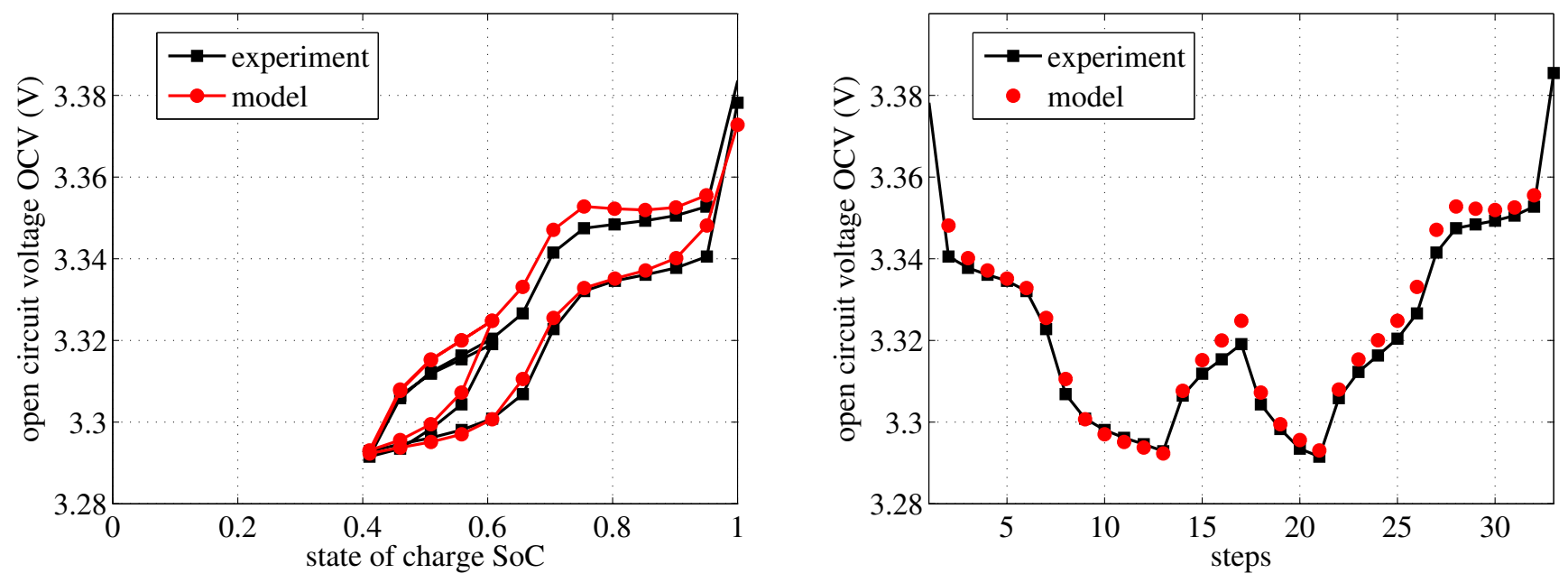

(c) Pulsed current test (for description see Fig. 2)

Fig. 8. Left: open circuit voltage OCV with respect to cell SoC. Right: Fictitious evolution of open circuit voltage OCV. 
open circuit if $i=0$, since its value is inversely proportional to the absolute value of cell current $|i|$. This satisfies the hypothesis 2 on the hysteresis model.

\section{NumeriCAL RESUltS}

The modified model is finally used to simulate the battery evolution, when the $\Delta$ SoC state of charge steps defined in the experiments are applied as a function of time. Three simulations are performed with the same charge variation profiles described in the three PC tests (a), (b), and (c). The simulations aim at showing the capability of the model of reproducing the experimental results for a $\mathrm{LiFePO}_{4}$ cell, i.e., assessing the accuracy of the model.

The simulation initial condition is $\mathrm{SoC}=1$, i.e., $Q_{0}=$ $20 \mathrm{~A} \mathrm{~h}$. The hysteresis parameter $\gamma$ is considered constant and is calculated as a fitting parameter by minimising the Root Mean Square Error (RMSE) between the simulation results and the experimental ones. Its value is $\gamma=17.45$.

Fig. 8 shows the simulation results in the SoC - OCV plane (left column) for the three cases investigated. The results are also represented on the right column graphs as a fictitious time evolution of the OCV, where the voltage values are determined every SoC step. Both simulated and experimental data are reported. It is important to note the excellent agreement between the experimental and the modelsimulated data, which follow very well the measured data. The overall RMSE is $5.3 \%$, while the maximum voltage error is $25 \mathrm{mV}$. This result in an error less than $1 \%$, if normalised to the maximum voltage value of $3.53 \mathrm{~V}$. The important result obtained is that the modified battery model is able to take into account the hysteresis phenomena with high fidelity. The model is simple and relies on a single parameter estimated by fitting.

\section{CONCLUSION}

We have shown in this paper the experimental characterisation of a $20 \mathrm{~A}$ h lithium-iron-phosphate $\left(\mathrm{LiFePO}_{4}\right)$ cell at room temperature, aimed at investigating its pronounced hysteresis behaviour. A circuit model is then proposed that accounts for the hysteresis effects in a very simple way. It is assumed that the voltage hysteresis measured on the Open Circuit Voltage $(\mathrm{OCV})$ is a dynamic function of the cell State-of-Charge (SoC) that can be described by a first order model. The hysteresis model "charge constant" parameter depends on the cell current and is identified by minimising the root mean square error of the simulations fitted on the experimental results.

The model looks simple and suitable for online battery state of charge and parameter estimation, but its accuracy still needs to be improved, as the rms error obtained is around $5 \%$. The results are very promising and further efforts will be devoted to improve the accuracy of the model.

\section{ACKNOWLEDGMENT}

This work is supported in part by University of Salerno FARB funds.

\section{REFERENCES}

[1] S. Lukic, R. Bansal, F. Rodriguez, and A. Emadi, "Energy Storage Systems for Automotive Applications," IEEE Transactions on Industrial Electronics, vol. 55, no. 6, pp. 2258-2267, Jun. 2008.

[2] S. Vazquez, S. M. Lukic, E. Galvan, L. G. Franquelo, and J. M. Carrasco, "Energy Storage Systems for Transport and Grid Applications," IEEE Transactions on Industrial Electronics, vol. 57, no. 12, pp. 3881-3895, 2010.

[3] M. S. Whittingham, "History, Evolution, and Future Status of Energy Storage," Proceedings of the IEEE, vol. 100, no. Special Centennial Issue, pp. 1518-1534, May 2012.

[4] M. A. Roscher, O. Bohlen, and J. Vetter, "OCV Hysteresis in Li-Ion Batteries including Two-Phase Transition Materials," International Journal of Electrochemistry, vol. 2011, pp. 1-6, 2011.

[5] M. A. Roscher and D. U. Sauer, "Dynamic electric behavior and open-circuit-voltage modeling of LiFePO4-based lithium ion secondary batteries," Journal of Power Sources, vol. 196, no. 1, pp. 331-336, 2011.

[6] H. Rahimi Eichi and M.-Y. Chow, "Modeling and analysis of battery hysteresis effects," in 2012 IEEE Energy Conversion Congress and Exposition (ECCE). IEEE, Sep. 2012, pp. 4479-4486.

[7] H. Rahimi-Eichi, U. Ojha, F. Baronti, and M.-Y. Chow, "Battery Management System: An Overview of Its Application in the Smart Grid and Electric Vehicles," IEEE Industrial Electronics Magazine, vol. 7, no. 2, pp. 4-16, Jun. 2013.

[8] K. W. E. Cheng, B. P. Divakar, H. Wu, K. Ding, and H. F Ho, "Battery-Management System (BMS) and SOC Development for Electrical Vehicles," IEEE Transactions on Vehicular Technology, vol. 60 , no. 1 , pp. 76-88, Jan. 2011.

[9] M. Brandl, H. Gall, M. Wenger, V. Lorentz, M. Giegerich, F. Baronti, G. Fantechi, L. Fanucci, R. Roncella, R. Saletti, S. Saponara, A. Thaler, M. Cifrain, and W. Prochazka, "Batteries and battery management systems for electric vehicles," in 2012 Design, Automation \& Test in Europe Conference Exhibition (DATE), Dresden, 2012, pp. 971-976.

[10] F. Baronti, G. Fantechi, E. Leonardi, R. Roncella, and R. Saletti, "Hierarchical platform for monitoring, managing and charge balancing of LiPo batteries," in 2011 IEEE Vehicle Power and Propulsion Conference (VPPC). Chicago: IEEE, Sep. 2011, pp. 1-6.

[11] H. Rahimi-Eichi, F. Baronti, and M.-Y. Chow, "Modeling and online parameter identification of Li-Polymer battery cells for SOC estimation," in 2012 IEEE International Symposium on Industrial Electronics. IEEE, May 2012, pp. 1336-1341.

[12] R. Restaino and W. Zamboni, "Comparing particle filter and extended kalman filter for battery State-Of-Charge estimation," in IECON 2012 - 38th Annual Conference on IEEE Industrial Electronics Society. IEEE, Oct. 2012, pp. 4018-4023.

[13] F. Baronti, W. Zamboni, N. Femia, R. Roncella, S. Rosi, S. Roberto, H. Rahimi Eichi, and M.-Y. Chow, "Parameter Identification of LiPo batteries in electric vehicles: a comparative study," in 2013 IEEE International Symposium on Industrial Electronics, Sep. 2013, pp. 1-7.

[14] M. A. Roscher, O. S. Bohlen, and D. U. Sauer, "Reliable State Estimation of Multicell Lithium-Ion Battery Systems," IEEE Transactions on Energy Conversion, vol. 26, no. 3, pp. 737-743, Sep. 2011.

[15] G. L. Plett, "Extended Kalman filtering for battery management systems of LiPB-based HEV battery packs: Part 2. Modeling and identification," Journal of Power Sources, vol. 134, no. 2, pp. 262-276, 2004.

[16] M. Chen and G. Rincon-Mora, "Accurate Electrical Battery Model Capable of Predicting Runtime and I-V Performance," IEEE Transactions on Energy Conversion, vol. 21, no. 2, pp. 504-511, Jun. 2006. 